\title{
Modelo de planeación y control de la producción a mediano plazo para una industria textil en un ambiente make to order ${ }^{*}$
}

\author{
Gerson Arredondo Ortega $a^{* *}$ \\ Kelly Vanessa Ocampo Jaramillo** \\ Juan Pablo Orejuela Cabrera**** \\ Carlos Alberto Rojas Trejos ${ }^{* * * * *}$
}

Recibido: 19/10/2015 - Aceptado: 08/08/2016

DOI: 10.22395/rium.v16n30a9

\begin{abstract}
Resumen
En el presente artículo se propone una metodología de planeación y control de la producción a mediano plazo para una industria del sector textil en un ambiente make to order. La metodología se basa en dos fases. En la primera fase se desarrolla un modelo de planeación agregada, que permite calcular las cantidades a producir en el taller de confección y la cantidad de prendas a maquilar, de tal forma que se maximice el beneficio total para la industria en el horizonte de planeación. En la segunda fase se plantea un modelo de programación de operaciones cuyo objetivo es secuenciar las cantidades a fabricar en el taller, de tal forma que se minimice el make span. Las dos fases propuestas constituyen una importante herramienta para apoyar la toma de decisiones sobre la gestión de la producción en industrias del sector textil.
\end{abstract}

Palabras clave: make to order, planeación agregada, programación de operaciones, makespan

El artículo se deriva del proyecto Propuesta Metodológica de Planeación y Control de la Producción a Mediano Plazo para una empresa del sector Textil en un ambiente Make to Order, ejecutado entre febrero de 2013 y junio de 2014 desde el programa de ingeniería industrial en la Universidad del Valle en Colombia. Financiación Universidad del Valle.

** Ingeniero Industrial, Universidad del Valle. Cargo: Ingeniero Industrial, Nestlé de Colombia S.A. Dirección de Contacto: Carrera 2, Calle 2, Bugalagrande, Valle del Cauca, Colombia. Correo lectrónico: gerson.arredondo@, correounivalle.edu.co.

*** Ingeniera Industrial, Universidad del Valle. Cargo: Ingeniera Industrial, Universidad del Valle. Dirección de Contacto: Cl. 14 \# 7-134, Zarzal, Valle del Cauca, Colombia. Correo electrónico: kelly.ocampo@correounivalle.edu.co

**** Ingeniero Industrial, Universidad del Valle. Magíster en Ingeniería con Énfasis en Ingeniería Industrial. Cargo: Docente Tiempo completo, Universidad del Valle, Cali, Colombia. Dirección de Contacto: Calle 13 \# 100-00, Ed 357, Cali, Valle del Cauca, Colombia. Correo electrónico: juan.orejuela@correounivalle.edu.co. Grupo de Investigación:Logistica y producción, Universidad del Valle.

***** Ingeniero Industrial, Universidad del Valle. Magíster en Ingeniería con Énfasis en Ingeniería Industrial. Cargo: Docente Programa Académico de Ingeniería Industrial, Universidad del Valle, Colombia. Dirección de Contacto: Calle 14 \# 7-134, Zarzal, Valle del Cauca, Colombia. Correo electrónico: carlos.rojas.t@correounivalle.edu.co. 


\title{
Planning and production control in the medium term for textile industry model in a make to order environment
}

\begin{abstract}
In this paper a methodology for planning and control of production in the medium term is proposed for the textile industry sector in a make to order environment. The methodology is based on two phases arising from the characteristics of the production system. In the first phase, an aggregate planning model, which allows the calculation of quantities produced in the own workshop and the amount to outsource of clothes to work on so that it meets the economic objectives and obtain the greatest benefit for the industry in the planning horizon. In the second stage, an operations programming model is proposed, which aims at producing sequences for the quantities that need to be produced in the workshop so that the make span is reduced. The two proposed phases constitute an important tool to support decision-making on production management in the textile sector.
\end{abstract}

Key words: make to order, aggregate planning, scheduling, make span. 


\section{INTRODUCCIÓN}

La planeación y el control de la producción proporcionan un marco de referencia en la toma de decisiones empresariales y se encargan de la conexión entre las estrategias organizacionales y las estrategias de operación de la empresa. Los alcances, la complejidad y las implicaciones de la planeación y control de la producción son influenciados por el ambiente de producción [1]. Los dos ambientes más conocidos son fabricación contra inventario y fabricación contra pedido; cada una de ellos plantea características que hacen que el proceso de planeación requiera un tratamiento diferente.

Las empresas que trabajan con una configuración contra inventario, por lo general, tiene sus instalaciones situadas en línea, y sus operaciones son repetitivas. En este ambiente el aprovechamiento adecuado de las instalaciones y de los recursos es determinado por la calidad del diseño del proceso inicial: si este es adecuado, el inventario de productos en curso será el mínimo posible, con lo que las actividades de programación se reducen a ajustar el ritmo de producción o el número de horas de funcionamiento de la cadena. Ambas actividades van a determinar el volumen de producción [2].

Por otro lado, están las empresas que fabrican bajo pedido, las cuales se caracterizan por fabricar una amplia variedad de productos en lotes pequeños, utilizando una distribución funcional, donde los lotes fluyen de un centro de trabajo a otro, con una secuencia de fabricación que varía con relación a los requerimientos del cliente y a las características del producto. El proceso de producción únicamente inicia después de que se recibe una orden de pedido por parte del comprador. En este ambiente el proceso de planeación y control de la producción resulta ser particularmente complejo, por la variedad y cantidad de decisiones que están involucradas.

Con base en lo anterior, surge la necesidad de definir adecuadamente la metodología de planeación de la producción que involucre las características del ambiente de producción y que permita un adecuado manejo de los recursos, de tal forma que contribuya en un incremento de productividad, la mejora en la calidad del producto y el cumplimiento de los tiempos de entrega.

En este orden de ideas, en la presente investigación se diseña una metodología de planeación de la producción a mediano plazo en un ambiente contra pedido, que permita determinar las cantidades a producir internamente y cantidades a producir por parte de terceros; asímismo, la secuencia de fabricación para cada orden de trabajo, con el propósito maximizar beneficio económico y mejorar el nivel de respuesta para la compañía. 


\section{AMBIENTES MAKE TO ORDER}

Existen numerosas investigaciones en las que se aborda el problema de planeación y control de la producción en los ambientes de fabricación contra pedido; algunas de estas se relacionan a continuación.

En [4] se presenta un marco unificado para la planeación y programación de la producción de un sistema de producción make to order. Los autores presentan una visión general de los principales conceptos y métodos para dar solución al problema de la planeación jerárquica de la producción, donde se platea que el problema se puede solucionar desde el enfoque basado en proyectos. La propuesta involucra el control de la carga y la relación entre los niveles jerárquicos de decisión del mediado y el corto plazo.

Por otra parte, [5] en búsqueda de un sistema de planeación y control de la producción que satisfaga todos los requerimientos de las industrias que operan bajo pedido, se realiza un rediseño del sistema basado en las tecnologías de la información y se presentan estas como una solución idónea al problema.

También [6] presentan una propuesta metodológica que inicia con la agrupación de factores de proceso, la aplicación de tecnología de grupos (TG) , la determinación de la capacidad instalada y requerida, finalizando con una propuesta de programación de la producción basada en la explotación del cuello de botella que permite definir las fechas de entrega de los pedidos.

Una propuesta metodológica para estimar la capacidad de producción de una empresa de servicios es desarrollada en [7], donde inicialmente se determina el recurso cuello de botella, para calcular la capacidad de los factores utilizando las fórmulas de capacidad intrínseca. El modelo busca maximizar los productos servidos en un periodo de tiempo donde incluye restricciones de instalaciones, en servicio, en recursos y generales. Para el caso de estudio se obtuvo como resultado que la capacidad es variable, ya que depende de una gran cantidad de factores presentados en la investigación.

El diseño de una metodología para planear y controlar la producción en un ambiente make to order en una empresa del sector manufacturero es presentado por [8], en la que se emplea un enfoque de multi project scheduling con restricciones de recursos. La propuesta se desarrolló empleando modelación matemática a partir de técnicas de programación por restricciones, donde se integran diferentes componentes como las relaciones de precedencia y las restricciones de recursos. La finalidad del modelo es configurar las actividades que componen cada proyecto $\mathrm{u}$ orden de producción teniendo en cuenta el consumo de recursos (máquinas y equipo) y las restricciones de entrega y de liberación de estos, con el fin de minimizar la fecha de entrega de todos los proyectos. 
En [9] se desarrolla una metodología que incluye un modelo metaheurístico basado en algoritmos genéticos multiobjetivo (elitist non-dominated sorting genetic algorithm, NSGA-II) en un ambiente de producción Job Shop. Esto, con el fin de minimizar tres variables fundamentales del proceso: tiempo total de proceso, costo de energía y accidentalidad laboral. Con la aplicación de la metodología, se logra encontrar resultados para las variables objeto de estudio, mejores un $42 \%$, respecto a las técnicas tradicionales de programación de la producción.

Finalmente, [10] presentan la aplicación de una nueva metodología para la programación de producción en una planta industrial, en la cual se combinan los ambientes fabricación para inventario (MTS) y fabricación bajo pedido (MTO). Esto implica responder a objetivos diferentes y, en ocasiones, opuestos, a partir de recursos limitados, con el consecuente impacto en el nivel de servicio. El estudio permite concluir que es posible para empresas que operan bajo entornos de producción híbridos, lograr equilibrio entre la respuesta al mercado y la utilización de los recursos disponibles.

\section{MATERIALES Y MÉTODOS}

Con base en la revisión bibliográfica expuesta, mediante la identificación y el análisis de las características propias de las diferentes metodologías de planeación y control de la producción y su funcionamiento en un ambiente de fabricación Make to Order, se presenta la siguiente metodología (ver figura 1).

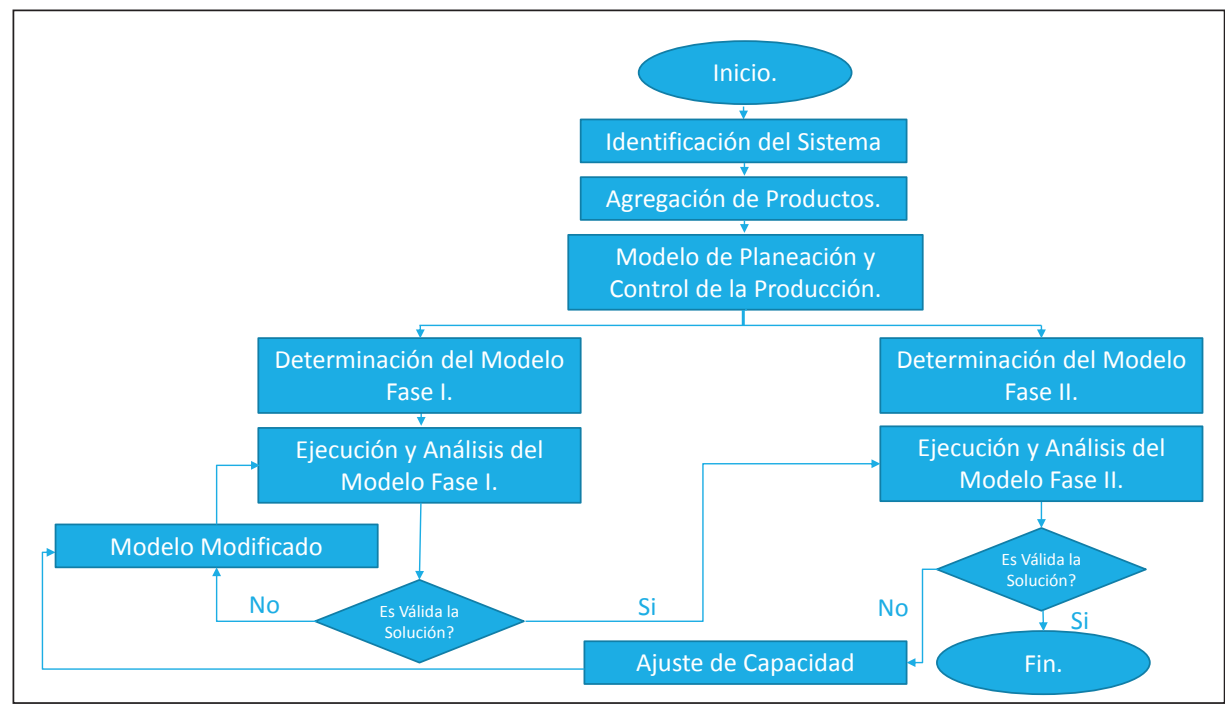

Figura 1. Flujograma propuesta metodológica.

Fuente: elaboración propia 


\subsection{Identificación del sistema}

En la primera actividad que concierne a la identificación o caracterización del sistema de fabricación se estableció que la empresa caso de estudio posee una demanda determinística, ya que la demanda mensual de cada producto en un mes determinado permite conocer con anterioridad la del mes anterior.

Los productos obedecen a una misma secuencia de fabricación, con unas variantes mínimas, donde si una prenda no es procesada en un recurso, a este se le asigna un tiempo de procesamiento igual a cero; se puede determinar por lo tanto que la empresa posee un sistema flow shop. También se maneja un lead time constante, ya que la empresa objeto de estudio se compromete a entregar los pedidos en un tiempo límite de un mes sin considerar la cantidad del mismo.

\subsection{Agregación de productos}

La empresa objeto de estudio ofrece a sus clientes un catálogo de 58 productos disponibles. Dada la gran cantidad de productos de los cuales muchos de ellos presentan características similares, se hace necesario implementar una metodología de agrupación que establezca familias de productos que compartan entre sí características definidas, con el objetivo de hacer más amigable el tratamiento de la información.

Creación de familias por secuencia de fabricación. Analizando la cantidad de ítems (58 productos) se encontró que dentro de ellos existían productos que llevaban el mismo proceso de fabricación. Para la creación del primer grupo de familias se desarrolló un análisis fundamentado en tecnología de grupos utilizando la metodología de inspección visual, la cual consiste en conformar las familias de productos mediante la revisión visual de la secuencia de fabricación e identificación de los coeficientes de similaridad. Estos se calculan sobre caracteres de dos estados, que registran "1", cuando el producto pasa por el proceso, y " 0 ", de lo contrario. El análisis dio como resultado la creación de tres familias.

Creación de familias por similaridad de forma. Sobre la base de las tres familias obtenidas en el paso anterior, se procedió a realizar una segunda agrupación, esta vez bajo el criterio de similaridad de forma, es decir, se agruparon aquellas prendas que comparten igual forma física, dando paso a un segundo grupo de familias en la que los productos, además de compartir la misma secuencia de fabricación, también compartían características físicas similares. De este análisis se obtuvo como resultado la creación de ocho familias.

Creación de familias por tiempo de procesamiento. En este paso se analizaron los tiempos de procesamiento en cada centro de trabajo para cada uno de los productos 
pertenecientes a las ocho familias obtenidas en el paso anterior. Se formaron entonces nuevas familias con aquellos productos que tenían tiempos similares en cada uno de los centros de trabajo. Como resultado se obtuvieron 28 familias.

Creación de familias por costos de producción y precio de venta. Observando las 28 nuevas familias obtenidas se encontró que los productos que conformaban cada una de ellas también compartían costos de producción y precio de venta similar. Con este último filtro se garantiza que las familias aquí obtenidas se adapten a los requerimientos de la metodología planteada. Como resultado de la agrupación se pasó de tratar con 58 productos a 28 familias. El proceso anterior se resume en la figura 2.

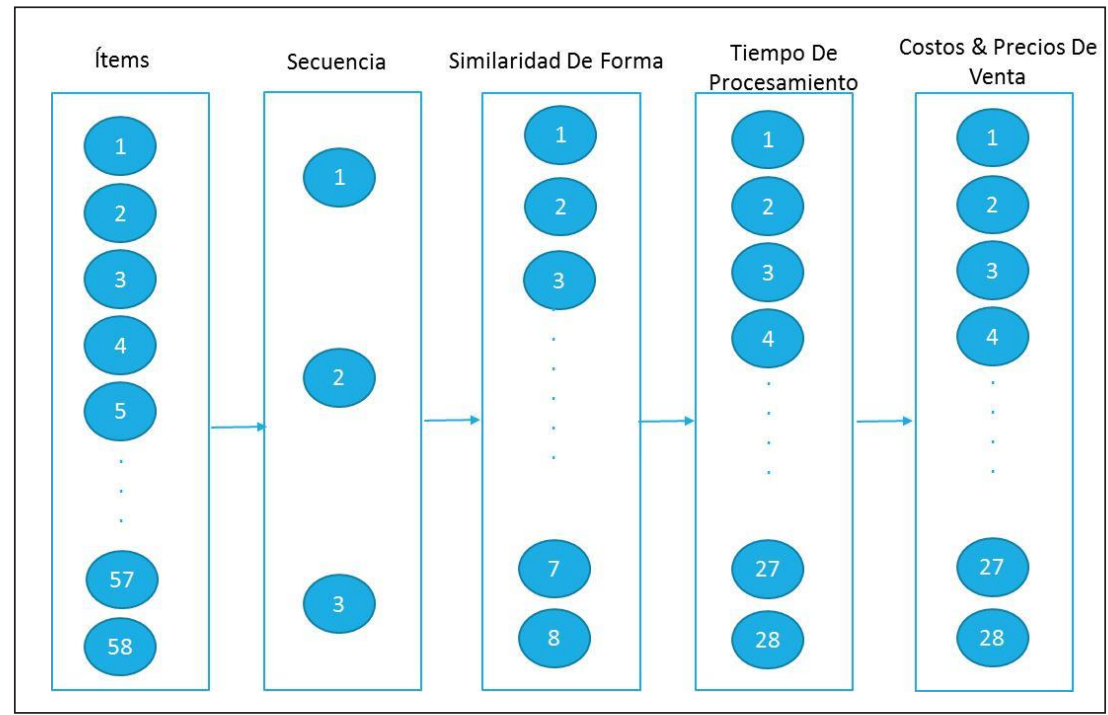

Figura 2. Familias de productos. Fuente: elaboración propia

\subsection{Determinación del modelo fase I. Plan agregado}

El modelo de planeación y control de la producción consta de dos fases, donde la información de salida del modelo fase I será información de entrada para el modelo fase II. A continuación, se describen cada una de ellas.

En la fase I se desarrolló un modelo de programación lineal entera mixto de planeación agregada, el cual tendrá como objetivo maximizar el beneficio recibido por la fabricación y venta de ítems en un horizonte de tiempo definido, considerando restricciones de capacidad, consumo de centro de trabajos y demanda. Finalmente, se determinarán las cantidades óptimas de la familia a fabricar en la organización en minutos ordinarios y extras; asímismo, la cantidad a enviar a terceros cuando la demanda exceda la capacidad de producción. 


\section{Formulación del modelo fase I}

El modelo se considera monoperíodo, mutiptoducto, con capacidad y demanda $\left(\mathrm{D}_{\mathrm{ji}}\right)$ determinística. Se tienen MOD conjunto de familias ( $i \in\{1,2,3, \ldots, n\}$ ), OP conjunto de órdenes de pedido $(\mathrm{j} \in\{1,2,3, \ldots, \mathrm{m}\})$, CT conjunto de centros de trabajo $(\mathrm{r} \in\{1,2,3, \ldots$, p\}), órdenes de pedido asociados a la familia i (OPMOD[i] whitin OP).

En este modelo, además, se tienen las siguientes consideraciones:

- Los tiempos de procesamiento para cada prenda pertenecientes a una familia son determinísticos.

- Los costos de producción y los precios de venta para cada una de las familias son determinísticos.

- El modelo se considera mono-período enmarcado en un horizonte fijo de planeación; es decir, solo se planea un periodo a la vez.

- No se consideran faltantes ni ventas perdidas.

Para la formulación del modelo fue necesario definir los parámetros descritos en la tabla 1 y las variables de decisión.

Tabla 1. Parámetros del modelo fase I

\begin{tabular}{|c|c|}
\hline Parámetro & Definición \\
\hline $\mathrm{D}_{\mathrm{ji}}$ & Demanda de la familia "i", de la orden "j". \\
\hline $\mathrm{TR}_{\mathrm{ir}}$ & Tiempo requerido para la fabricación de la familia "i”" en el centro de trabajo " $r$ ". \\
\hline $\mathrm{TO}_{\mathrm{r}}$ & $\begin{array}{l}\text { Tiempo total disponible del centro de trabajo " } \mathrm{r} \text { " en minutos ordinarios (Tiempo disponible } \\
\text { en un mes por el número de recursos en el centro r). }\end{array}$ \\
\hline $\mathrm{TE}_{\mathrm{r}}$ & $\begin{array}{l}\text { Tiempo total disponible del centro de trabajo "r" en minutos extras (Tiempo disponible en un } \\
\text { mes por el número de recursos en el centro r) }\end{array}$ \\
\hline $\mathrm{CO}_{\mathrm{i}}$ & Costo de producir una prenda de la familia "i" en minutos ordinarios \\
\hline $\mathrm{CE}_{\mathrm{i}}$ & Costo de producir una prenda de la familia "i”" en minutos extras \\
\hline $\mathrm{CM}_{\mathrm{i}}$ & Costo de producir una prenda de la familia "i”" enviada a maquila \\
\hline $\mathrm{HO}_{\mathrm{r}}$ & Minutos ordinarios que aporta un empleado en el centro de trabajo "r" en el horizonte de tiempo \\
\hline $\mathrm{HE}_{\mathrm{r}}$ & Minutos extras que aporta un empleado en el centro de trabajo " $\mathrm{r}$ " en el horizonte de tiempo \\
\hline CFM & Costos fijos de transporte incurridos cuando se envían prendas a maquilar \\
\hline $\mathrm{CFO}$ & Costo fijo de contratación \\
\hline $\mathrm{PV}_{\mathrm{i}}$ & Precio de venta de la prenda asociada a la familia "i" \\
\hline
\end{tabular}

Fuente: elaboración propia 


\section{Variables de decisión}

$Q O_{j i}$ : cantidad de la familia “i”" de la orden “j”" a producir en el taller propio en minutos ordinarios.

$Q E_{j i}$ : cantidad de la familia " $\mathrm{i}$ ” de la orden “j”" a producir en el taller propio en minutos extras.

$Q M_{j i}$ : cantidad de la familia "i”" de la orden "j" a enviar a maquila.

$N P_{r}$ : cantidad de empleados a contratar en el centro " $r$ " en jornada de trabajo ordinaria.

$Y$ : $\quad$ variable binaria donde un valor de " 1 " indica que se maquila unidades y se asumen los costos fijos de flete a maquila y " 0 " de lo contrario.

\section{Modelo matemático fase I}

A continuación se presenta el modelo matemático correspondiente a la presente fase.

$$
\begin{aligned}
Z_{\max }= & \Sigma_{j i n O P} \Sigma_{i \in M O D O P[j]}\left(P V_{i}-C O_{i}\right) * Q O_{j i}+\Sigma_{j i n O P} \Sigma_{i \in M O D O P[j]}\left(P V_{i}-C E_{i}\right) * \\
& Q E_{j i}-\Sigma_{r \in C T} N P_{r} * C F O+\Sigma_{j i n O P} \Sigma_{i \in M O D O P[j]}\left(P V_{i}-C M_{i}\right) * Q M_{j i}-C F M * Y
\end{aligned}
$$

Sujeto a:

$$
\begin{gathered}
\Sigma_{j i n O P} \Sigma_{i \in M O D O P[j]} T R_{i r} * Q O_{j i} \leq N P_{r} * H O_{r} \forall r \in C T \\
N P_{r} * H O_{r} \leq T O_{r} \forall r \in C T \\
\Sigma_{j i n O P} \Sigma_{i \in M O D O P[j]} T R_{i r} * Q E_{j i} \leq N P_{r} * H E_{r} \forall r \in C T \\
N P_{r} * H E_{r} \leq T E_{r} \forall r \in C T \\
\Sigma_{j i n O P} \Sigma_{i \in M O D O P[j]} Q M_{j i} \geq M^{*} Y \\
Q O_{j i}+Q E_{j i}+Q M_{j i}=D_{j i} \forall i \in M O D, \forall j \in O P \\
Q O_{j i} \geq 0 \quad \forall i \in M O D, \forall j \in O P \\
Q E_{j i} \geq 0 \forall i \in M O D, \forall j \in O P \\
Q M_{j i} \geq 0 \forall i \in M O D, \forall j \in O P \\
Q E_{j i}, Q M_{j i} \in Z ; \forall i \in M O D, \forall j \in O P \\
Y \in\{0,1\}
\end{gathered}
$$


El objetivo del modelo es maximizar el beneficio total obtenido por la fabricación y venta de familia de producto (ecuación 1), encontrando la combinación óptima a producir bajo los tres escenarios posibles, es decir, cantidad a producir en minutos extras $\left(Q O_{j i}\right)$, cantidad a producir en minutos extras $\left(Q E_{j i}\right)$, y cantidad a enviar a maquila $\left(Q M_{j i}\right)$. Asímismo, el número de empleados a contratar en cada centro de trabajo $\left(N P_{r}\right)$, sujeto a restricciones de capacidad en minutos ordinarios y extras (ecuaciones 2 y 4 respetivamente), de mano de obra en minutos ordinarios y extras (ecuaciones 3 y 5 respectivamente), restricción que controla la subcontratación donde la variable binaria Y toma el valor de 1, si la variable $Q M_{j i}>0$ y se asume el costo fijo de flete; en caso contrario toma el valor de 0 (ecuación 6), la restricción de balance de demanda (ecuación 7) y por último las obvias (ecuaciones $8,9,10,11$ y 12).

\subsection{Determinación del modelo fase II. Scheduling}

Se formula un modelo matemático lineal entero mixto de Scheduling, que tiene como objetivo minimizar el tiempo máximo de terminación de todos los trabajos (make span), mediante la determinación de la secuencia óptima de fabricación de los trabajos, los cuales estarán representados por las familias de productos a fabricar en el taller propio con base en los minutos ordinarios disponibles, datos que son proporcionados por el modelo de la fase anterior. Para ello se utiliza como modelos de referencia los trabajos realizados por [3] y [11], ya que se ajustan a las características que presenta el sistema productivo de la empresa objeto de estudio.

\section{Formulación del modelo fase II}

Se considera la secuencia de fabricación para todos los trabajos determinística, y bajo el sistema flow shop, donde el conjunto de centros de trabajo está representado por MAQ y el conjunto de trabajos a programar por $T B$. Los trabajos están representados por las cantidades a producir en el taller propio en minutos ordinarios $\left(Q O_{j i}\right)$, donde tienen la misma prioridad dentro del sistema, y los tiempos de procesamiento son determinísticos. Se tienen, además, las siguientes consideraciones:

- Un centro de trabajo no podrá procesar más de un trabajo al tiempo.

- No se consideran tiempos de transporte, ni alistamiento.

- Un trabajo solamente se considerará terminado en el momento en que sus operaciones en cada centro de trabajo hayan sido terminadas totalmente.

Para la formulación del modelo se definieron los parámetros que se muestran en la tabla 2 y las variables de decisión correspondientes. 
Modelo de planeación y control de la producción a mediano plazo para una industria textil en un ambiente ... 179

Tabla 2. Parámetros del modelo fase II

\begin{tabular}{|c|l|}
\hline Parámetro & \multicolumn{1}{|c|}{ Definición } \\
\hline QTB & Cantidad de trabajos a programar en el Flowshop \\
\hline $\mathbf{Q M}$ & Cantidad de centros de trabajo en el Flowshop \\
\hline $\mathbf{T R}_{\mathrm{ij}}$ & Tiempo de proceso de la máquina i requerido para procesar el trabajo $\mathrm{j}$ \\
\hline
\end{tabular}

Fuente: elaboración propia

\section{Variables de decisión}

$\mathrm{X}_{\mathrm{jk}}$ : variable binaria que vale 1 si el trabajo " $\mathrm{j}$ " se programa en la secuencia " $\mathrm{k}$ " y 0 de lo contrario

$T O_{i k}$ : variable continua que indica el tiempo ocioso que experimenta el centro de trabajo "i" desde el momento en que termina de procesar el trabajo en la k-esima posición de la secuencia hasta el momento que inicia el trabajo en la posición $(\mathrm{k}+1)$-ésima de la secuencia

$T E_{i k}$ : variable continua que indica el tiempo que espera el trabajo en la k-ésima posición de la secuencia desde el momento que se termina la realización de la posición en el centro de trabajo "i" y el inicio de la operación siguiente en el centro de trabajo "i+1".

\section{Modelo matemático fase II}

A continuación, se presenta el modelo matemático correspondiente a la presente fase.

$$
Z_{\min }=\sum_{q \in\{1 . .(Q M-1)\}} \sum_{j \in T B} x_{j, 1} * T R_{i, j}+\sum_{j \in\{1 . .(Q T-1)\}} T O_{Q M, j}+\sum_{j \in T B} T R_{, Q M, j}
$$

Sujeto a:

$$
\begin{gathered}
\sum_{j \in T B} x_{j k}=1 \forall k \in\{1 . . Q T B\} \\
\sum_{k \in\{1 . . Q T B\}} x_{j k}=1 \forall j \in\{1 \ldots T B\} \\
T O_{i k}+\left[\sum_{j \in T B} x_{j,(k+1)} * T R_{i j}\right]+T E_{i,(k+1)}=T E_{i, k}+\left[\sum_{j \in T B} x_{j k} * T R_{(i+1), j}\right]+T O_{(i+1), k} \\
\forall k \in\{1 \ldots Q T B-1\} ; \forall i \in\{1 \ldots Q M-1\}
\end{gathered}
$$




$$
\begin{gathered}
T E_{i 1}=0 \forall i \in\{1 \ldots Q M-1\} \\
T Q_{1 k}=0 \forall k \in\{1 \ldots Q T B-1\} \\
T O_{i k} \geq 0 \forall i \in M A Q, k \in T B \\
T E_{i k} \geq 0 \forall i \in M A Q, k \in T B \\
X_{j k} \in\{0,1\} \forall j \in T B, k \in T B
\end{gathered}
$$

El objetivo del modelo es minimizar el tiempo máximo de terminación de todos los trabajos o make span (ecuación 13), sujeto a restricciones de secuencia tal como la ecuación (14) donde asegura que para cada posición de la secuencia debe haber un único trabajo; la ecuación (15) garantiza que un trabajo solo sea secuenciado una vez; la ecuación (16) define la relación que debe existir entre las variables físicas y funcionales del modelo $T O_{i k}, T E_{i k}$ y $X j_{k}$ (ver figura 3); la ecuación (17) asegura que el primer trabajo de la secuencia no espera $\left(T E_{i 1}=0\right)$; la ecuación (18) garantiza que el primer centro de trabajo del flow shop no experimenta tiempo ocioso y, por último, las ecuaciones o restricciones obvias $\left(19,20\right.$ y 21) si $T O_{i k}>0$, es decir, la máquina está un tiempo desocupada antes de empezar a procesar el trabajo en la posición $(k+1)$ ésima en la secuencia, lo anterior implica entonces que ese trabajo, el de la posición $(k+1)$-ésima, inició su proceso en la máquina i inmediatamente después de que dejó la máquina $i-1$; es decir, $T E_{(i-1)(k+1)}=0$. Lo anterior se explica, dado que si la máquina $i$ tuvo que esperar para procesar e iniciar el proceso del trabajo en la posición $k+1$ de la secuencia, era porque este trabajo no se había terminado en la máquina $i-1$; por tal motivo, al terminarse en esa máquina (i-1) y al estar ociosa la $i$, es natural que pase de una vez a procesar en la máquina $i$ (ver figura 3 ).

\subsection{Ajuste de capacidad}

Como el modelo de planeación propuesto en la fase 1 al ser en esencia un plan agregado no considera las precedencias, ni el tiempo que deben esperar los trabajos en cada centro de trabajo mientras que un trabajo está siendo procesado; por lo tanto, se deben hacer ajustes en el modelo de planeación para que el resultado del make span este cerca al valor esperado. Para esto se consideraron dos opciones: calcular el factor de utilización total y alterar los tiempos de proceso para cada familia en cada centro; y calcular el factor de utilización por centro de trabajo y alterar la restricción de capacidad en el taller propio en horas ordinarias propuesto por [2]. 


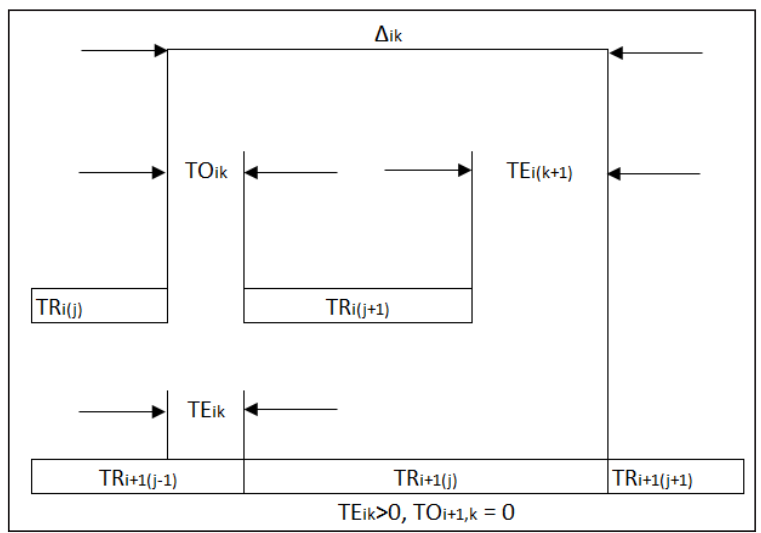

Figura 3. Relación entre las variables $\mathrm{TO}_{\mathrm{ik},} \mathrm{TE}_{\mathrm{ik}} \mathrm{y} \mathrm{X}_{\mathrm{jk}}$. Fuente: adaptado de [3] y [11]

Factor de Utilización $=\frac{\text { Tiempo Total Proceso de todos los Trabajos }}{\text { Minutos Disponibles }}$

Donde:

$$
\text { Minutos Disponibles }=\text { Makespan } * \mathrm{~N}^{\mathrm{o}} \text { total } \mathrm{CT}
$$

Por lo tanto, los tiempos de proceso para cada familia serán calculados con base con el factor de utilización total utilizando la siguiente fórmula:

$$
\text { Tiempos de Proceso }=\frac{\text { Tiempo Requerido Inicial }}{\text { Factor de Utilización }}
$$

Factor de Utilización por Cada Centro $=\frac{\text { Tiempo total de proceso por CT }}{\text { Makespan }}$

\section{CASO DE ESTUDIO}

La empresa objeto de estudio pertenece al sector confección-textil, dedicada exclusivamente a la fabricación de uniformes tipo industrial, la cual ofrece a sus clientes un portafolio de productos disponibles para su venta y solo en casos muy particulares los clientes definen el diseño y otros materiales a utilizar para la elaboración de las prendas. La organización hace recepción de los pedidos de sus clientes hasta el último día del mes previo a la realización del producto, es decir, de antemano se conoce la cantidad a producir en el siguiente mes; las órdenes presentan especificaciones y cantidades variables, generando así aspectos como flexibilidad y variedad de las características propias de un ambiente make to order. 
Los pedidos son comprometidos a entregar en un término fijo correspondiente a 30 días, contados a partir del primer día de cada mes, independiente del volumen a producir. Cuando los productos a elaborar en un mes determinado exceden la capacidad de producción del taller, se cuenta con la opción de tercerizar la elaboración del producto con el objetivo de cumplir con las fechas de entrega establecidas; a este proceso se le conoce como maquila.

La maquila consiste, entonces, en la elaboración de las prendas en talleres externos a la organización. Cuando se hace necesario recurrir a este proceso, la empresa entrega las materias primas a estos talleres, garantizando así la calidad del producto. Una vez terminado todo el proceso productivo en los talleres, estos retornan el producto terminado a la organización para completar el pedido y ser entregado al cliente en el plazo establecido.

El proceso productivo de la realización de las prendas pasa por diferentes etapas que, en el caso del modelo planteado, representan los centros de trabajo. A su vez, cada centro de trabajo involucra la utilización de maquinaria la cual será el recurso que limita la capacidad del mismo. Se debe aclarar que en el centro de trabajo la "revisión" del proceso se realiza de forma manual; para este caso, la mano de obra empleada en el proceso será el recurs limitante de la capacidad de dicho centro. Los diferentes centros de trabajos identificados, así como los recursos, su cantidad disponible y el número total de minutos disponibles para cada centro de trabajo en jornada ordinaria y jornada extra en un horizonte de tiempo mensual se muestran en la tabla 3.

Tabla 3. Capacidad de los centros de trabajo

\begin{tabular}{|l|l|l|c|c|c|}
\hline \multicolumn{2}{|c|}{ Centros de trabajo } & \multicolumn{1}{|c|}{ Recurso involucrado } & Cantidad & $\begin{array}{c}\text { Minutos } \\
\text { disponibles al } \\
\text { mes en cada } \\
\text { CT (Jornada } \\
\text { Ordinaria) }\end{array}$ & $\begin{array}{c}\text { Minutos } \\
\text { disponibles al } \\
\text { mes en cada } \\
\text { CT (Jornada } \\
\text { Extra) }\end{array}$ \\
\hline CT 1 & Corte & $\begin{array}{l}\text { Máquina de corte de tela tipo } \\
\text { industrial }\end{array}$ & 1 & 14664 & 6240 \\
\hline CT 2 & $\begin{array}{l}\text { Confección y } \\
\text { fileteado }\end{array}$ & $\begin{array}{l}\text { Máquina plana y fileteadora tipo } \\
\text { industrial }\end{array}$ & 18 & 263952 & 112320 \\
\hline CT 3 & $\begin{array}{l}\text { Ojalado y } \\
\text { botones }\end{array}$ & $\begin{array}{l}\text { Ojaladora e instaladora de } \\
\text { botones }\end{array}$ & 1 & 73320 & 6240 \\
\hline CT 4 & Revisión & Personal de revisión de prendas & 5 & 70200 & 31200 \\
\hline
\end{tabular}




\begin{tabular}{|c|c|c|c|c|c|}
\hline \multicolumn{2}{|c|}{ Centros de trabajo } & Recurso involucrado & Cantidad & $\begin{array}{c}\text { Minutos } \\
\text { disponibles al } \\
\text { mes en cada } \\
\text { CT (Jornada } \\
\text { Ordinaria) }\end{array}$ & $\begin{array}{c}\text { Minutos } \\
\text { disponibles al } \\
\text { mes en cada } \\
\text { CT (Jornada } \\
\text { Extra) }\end{array}$ \\
\hline CT 5 & Estampado & Máquina screen para estampados & 1 & 14664 & 6240 \\
\hline CT 6 & Empaque & Selladora & 1 & 14664 & 6240 \\
\hline
\end{tabular}

Fuente: elaboración propia

Para el cálculo del número total disponible en minutos para un horizonte de tiempo mensual (considerando un mes de 30 días) para cada centro de trabajo en jornada ordinaria, se asumió una jornada diaria de 9.4 horas/día por recurso en el centro de trabajo; dicha cantidad se multiplicó por el número de recursos existentes en el centro, se replicó a 26 días laborales al mes y se calculó su equivalencia en minutos. Al igual se calculó el número total de minutos extras disponibles en el taller propio, asumiendo un límite permitido de 4 minutos extra / día por recurso en cada centro.

Los tiempos de procesamiento unitario que requiere cada familia de productos en cada centro de trabajo se ilustran en la tabla 4. De igual forma se emplea el precio de venta unitario al público para cada tipo de familia de productos y el costo unitario de fabricación para cada familia, cuando esta es producida en minutos ordinarios, minutos extra o si es producto enviado a maquila; dicho costo está compuesto por el costo de materia prima y costos de mano de obra empleado en cada una de las tres modalidades.

Tabla 4. Tiempos de procesamiento en centros de trabajo

\begin{tabular}{|c|c|c|c|c|c|c|c|}
\hline \multicolumn{8}{|c|}{ Tiempo en minutos por unidad } \\
\hline \multirow{2}{*}{ Familias } & Corte & $\begin{array}{c}\text { Confec- } \\
\text { ción y } \\
\text { fileteado }\end{array}$ & $\begin{array}{c}\text { Ojalado y } \\
\text { botones }\end{array}$ & Revisión & $\begin{array}{c}\text { Estam- } \\
\text { pado y/o } \\
\text { bordado }\end{array}$ & Empaque & Total \\
\cline { 2 - 9 } & CT 1 & CT 2 & CT 3 & CT 4 & CT 5 & CT 6 & \\
\hline F1 & 1,35 & 10 & 0,5 & 0,5 & 2 & 0,5 & 14,85 \\
\hline F2 & 1,35 & 10 & 0,5 & 0,5 & 2 & 0,5 & 14,85 \\
\hline F3 & 1,35 & 10 & 0,5 & 0,5 & 2 & 0,5 & 14,85 \\
\hline F4 & 0,7 & 8 & 0 & 0,5 & 2 & 0,5 & 11 \\
\hline F5 & 0,7 & 8 & 0 & 0,5 & 2 & 0,5 & 11 \\
\hline
\end{tabular}


184 Gerson Arredondo Ortega - Kelly V. Ocampo Jaramillo - Juan P. Orejuela Cabrera - Carlos A. Rojas Trejos

\begin{tabular}{|c|c|c|c|c|c|c|c|}
\hline \multicolumn{8}{|c|}{ Tiempo en minutos por unidad } \\
\hline \multirow[t]{2}{*}{ Familias } & Corte & $\begin{array}{c}\text { Confec- } \\
\text { ción y } \\
\text { fileteado }\end{array}$ & $\begin{array}{c}\text { Ojalado y } \\
\text { botones }\end{array}$ & Revisión & $\begin{array}{l}\text { Estam- } \\
\text { pado y/o } \\
\text { bordado }\end{array}$ & Empaque & \multirow[t]{2}{*}{ Total } \\
\hline & CT 1 & CT 2 & CT 3 & CT 4 & CT 5 & CT 6 & \\
\hline F6 & 1,7 & 13 & 0,2 & 0,4 & 2 & 0,5 & 17,8 \\
\hline F7 & 1,7 & 13 & 0,2 & 0,4 & 2 & 0,5 & 17,8 \\
\hline F8 & 1,7 & 13 & 0,2 & 0,4 & 2 & 0,5 & 17,8 \\
\hline F9 & 1,2 & 13 & 0,2 & 0,4 & 2 & 0,5 & 17,3 \\
\hline F10 & 1,2 & 11 & 0,2 & 0,4 & 2 & 0,5 & 15,3 \\
\hline F11 & 1,2 & 11 & 0,2 & 0,4 & 2 & 0,5 & 15,3 \\
\hline F12 & 1,5 & 11 & 0,2 & 0,4 & 2 & 0,5 & 15,6 \\
\hline F13 & 1,6 & 11 & 0,2 & 0,4 & 2 & 0,5 & 15,7 \\
\hline F14 & 1,6 & 11 & 0,2 & 0,4 & 2 & 0,5 & 15,7 \\
\hline F15 & 2 & 15 & 0,5 & 0,5 & 2 & 0,5 & 20,5 \\
\hline F16 & 2 & 15 & 0,5 & 0,5 & 2 & 0,5 & 20,5 \\
\hline F17 & 2 & 15 & 0,5 & 0,5 & 2 & 0,5 & 20,5 \\
\hline F18 & 1,35 & 8 & 0,2 & 0,4 & 2 & 0,5 & 12,45 \\
\hline F19 & 1,5 & 8 & 0,2 & 0,4 & 2 & 0,5 & 12,6 \\
\hline $\mathrm{F} 20$ & 1,35 & 8 & 0,2 & 0,4 & 2 & 0,5 & 12,45 \\
\hline F21 & 1,35 & 8 & 0,2 & 0,4 & 2 & 0,5 & 12,45 \\
\hline F22 & 1 & 8 & 0,2 & 0,4 & 2 & 0,5 & 12,1 \\
\hline $\mathrm{F} 23$ & 1,7 & 10 & 0 & 0,4 & 2 & 0,5 & 14,6 \\
\hline $\mathrm{F} 24$ & 1,7 & 10 & 0 & 0,4 & 2 & 0,5 & 14,6 \\
\hline $\mathrm{F} 25$ & 1,7 & 10 & 0 & 0,4 & 2 & 0,5 & 14,6 \\
\hline F26 & 1,5 & 3 & 0 & 0,4 & 0 & 0,5 & 5,4 \\
\hline $\mathrm{F} 27$ & 0,5 & 3 & 0 & 0,4 & 0 & 0,5 & 4,4 \\
\hline F28 & 0,3 & 3 & 0 & 0,4 & 0 & 0,5 & 4,2 \\
\hline
\end{tabular}

Fuente: elaboración propia

Para el desarrollo de la metodología aquí planteada se escogió la demanda correspondiente a un mes específico. Este período fue elegido de forma aleatoria y en este se 
considera que la empresa operó bajo condiciones normales. Los datos correspondientes a las órdenes de pedido, las familias que integran cada orden y su volumen para el mes correspondiente se presentan en la tabla 5 .

Tabla 5. Pedidos consolidados

\begin{tabular}{|c|c|c|c|}
\hline Orden & Prendas & Familia perteneciente & Cantidad \\
\hline 1 & Camisa dril & $\mathrm{F} 2$ & 3 \\
\hline 2 & Pantalón enresortado dril blanco & F20 & 5 \\
\hline \multirow{5}{*}{3} & Cofia enresortado dacrón holandés & F26 & 3000 \\
\hline & Pantalón enresortado dril blanco & F10 & 4161 \\
\hline & Camibuso en dacrón & F25 & 824 \\
\hline & Delantal jardinero dril & $\mathrm{F} 22$ & 2530 \\
\hline & Camisa dacrón & F1 & 2 \\
\hline 4 & Camibuso en dacrón & F25 & 75 \\
\hline \multirow{2}{*}{5} & Delantal lab. M/c dacrón & F21 & 2 \\
\hline & Delantal lab. M/c cierre & F20 & 4 \\
\hline \multirow{6}{*}{6} & Camibuso en dril & $\mathrm{F} 23$ & 1837 \\
\hline & Delantal lab. M/c dacrón & F20 & 30 \\
\hline & Pantalón parche & F14 & 1923 \\
\hline & Tapaboca en dacrón & F28 & 1000 \\
\hline & Cofia enresortada en malla y velo & F27 & 780 \\
\hline & Delantal jardinero dril & F22 & 1100 \\
\hline \multirow{6}{*}{7} & Pantalón enresortado dril blanco & F10 & 144 \\
\hline & Camisa dacrón & F2 & 106 \\
\hline & Delantal lab. M/c cierre & F21 & 2 \\
\hline & Delantal lab. M/c dacrón & F20 & 3 \\
\hline & Camisa dril & $\mathrm{F} 2$ & 5 \\
\hline & Camisa dacrón & F1 & 8 \\
\hline \multirow{3}{*}{8} & Camibuso en dacrón & F25 & 171 \\
\hline & Pantalón enresortado dril blanco & F10 & 165 \\
\hline & Cofia enresortada dacrón holandés & F26 & 50 \\
\hline
\end{tabular}

Fuente: elaboración propia 


\section{RESULTADOS Y DISCUSIÓN}

Para la ejecución y análisis de los resultados del modelo se utilizó la demanda de un mes consolidado y los respectivos valores de los parámetros expuestos en el apartado anterior, asociados a la empresa objeto de estudio. Los modelos fueron programados en AMPL (A modeling language for mathematical programming) donde se empleó el Solver Gurobi a través de la Plataforma Neos Server.

\subsection{Análisis de resultados}

$\mathrm{Al}$ resolver el modelo con los datos recopilados en el caso de estudio se obtuvo un beneficio máximo de $\$ 115.570 .605,60$, donde las cantidades óptimas a fabricar fueron $11.162,3120$ y 3648 prendas para minutos ordinarios $\left(Q O_{j i}\right)$, minutos extras $\left(Q E_{j i}\right)$ y maquila $\left(Q M_{j i}\right)$, respectivamente. Una vez conocidas las cantidades óptimas a fabricar, se procedió a analizar el consumo de recursos por centro de trabajo para el taller propio durante la jornada de trabajo ordinaria y jornada extra. Los resultados obtenidos se especifican en la tabla 6.

Tabla 6. Consumo recursos centro de trabajo

\begin{tabular}{|l|c|c|c|}
\hline \multicolumn{1}{|c|}{ Centros de trabajo } & $\begin{array}{c}\text { Operarios a } \\
\text { llamar en cada } \\
\text { centro } \mathrm{NPr}\end{array}$ & $\begin{array}{c}\text { \% Utilización del centro } \\
\text { de trabajo en minutos } \\
\text { ordinarios }\end{array}$ & $\begin{array}{c}\text { \% Utilización del centro } \\
\text { de trabajo en minutos } \\
\text { extras }\end{array}$ \\
\hline CT1 - Corte & 1 & $99,998 \%$ & $78,45 \%$ \\
\hline CT2 - Confección y fileteado & 6 & $34,183 \%$ & $26,82 \%$ \\
\hline CT3-Ojalado y botones & 2 & $46,993 \%$ & $12,47 \%$ \\
\hline CT4 - Revisión & 1 & $25,224 \%$ & $20,00 \%$ \\
\hline CT5 - Estampado & 1 & $100,000 \%$ & $100,00 \%$ \\
\hline CT6 - Empaque & 1 & $38,059 \%$ & $25,00 \%$ \\
\hline
\end{tabular}

Fuente: elaboración propia

Como se evidencia en la tabla 10 los centros de trabajo con mayor porcentaje de utilización de su capacidad en minutos ordinarios son corte (CT1) y estampado (CT5), cada uno de ellos con una utilización del $99.998 \%$ y 100\%, respectivamente, seguidos de ojalado y botones (CT3), empaque (CT6), confección y fileteado (CT2)y revisión (CT4), cada uno con un porcentaje de utilización del 46.993\%, 38.059\%, 34.183\% y $25.224 \%$, respectivamente. Al igual que en minutos ordinarios, los centros de trabajo de mayor porcentaje de utilización de su capacidad en minutos extras son estampado (CT5), seguido de corte (CT1) con una utilización del 100\% y 78.45\%, respectivamente, 
seguidos de confección y fileteado (CT2), empaque (CT6), revisión (CT4) y ojalado y botones (CT3) cada uno con un porcentaje de utilización del 26,82\%, 25\%, 20\% y $12.47 \%$, respectivamente. Para el caso del centro de trabajo ojalado y botones (CT3) las familias asignadas por el modelo a realizar en minutos extras no pasan por dicho centro, por ende este recurso nunca se ocupa.

\subsection{Análisis de sensibilidad modelo fase I}

Para analizar los resultados iniciales del modelo se realizó un análisis de sensibilidad, haciendo variar los parámetros más críticos del mismo. Dentro de los parámetros evaluados están: la demanda $\left(D_{j i}\right)$ y la capacidad de los centros de trabajo del taller $\left(T O_{r}\right.$ y $\left.T E_{r}\right)$.

\subsubsection{Variación en la demanda}

En este caso se aumentó la demanda en 50\%, 100\%, 150\%, 200\%, con el propósito de ver el impacto de las modificaciones en la función objetivo y en las variables de interés. Se encontró que con dichas modificaciones en la demanda, el impacto en la función objetivo $(F O)$ con variaciones de 50\%, 100\%, 150\% y 200\% es creciente, pero no proporcional, ya que con un incremento del $50 \%$ se genera un incremento de la función objetivo en 37\%, mientras que con un incremento del 150\%, la función objetivo mejora en un $103 \%$ con relación a la obtenida en el caso inicial (caso 0) (ver tabla 7).

Tabla 7. Incremento porcentual demanda vs. beneficio total

\begin{tabular}{|c|c|c|c|}
\hline Casos & Demanda & Beneficio total & Porcentaje no obtenido \\
\hline Caso 0 & $0 \%$ & $0 \%$ & $0 \%$ \\
\hline Caso 1 & $50 \%$ & $37 \%$ & $13 \%$ \\
\hline caso 2 & $100 \%$ & $71 \%$ & $29 \%$ \\
\hline Caso 3 & $150 \%$ & $104 \%$ & $46 \%$ \\
\hline Caso 4 & $200 \%$ & $134 \%$ & $66 \%$ \\
\hline
\end{tabular}

Fuente: elaboración propia

Es importante mencionar que con la variación del $37 \%$ se presenta el mayor aumento en el valor de la función objetivo respecto al caso inicial; es decir, la función objetivo se incrementa en un valor cercano al porcentaje de variación de la demanda (ver tabla 8). 
Tabla 8. Incremento demanda vs. beneficio total

\begin{tabular}{|c|c|c|c|}
\hline Escenarios & Demanda & Beneficio total & \multicolumn{2}{|c|}{ Crecimiento } \\
\hline Caso 0 & 17930 & $\$ 115.570 .605,60$ & 0 \\
\hline Caso 1 & 26900 & $\$ 158.430 .945,40$ & $\$ 42.860 .339,80$ \\
\hline Caso 2 & 35860 & $\$ 197.906 .359,20$ & $\$ 82.335 .753,60$ \\
\hline Caso 3 & 44331 & $\$ 235.359 .988,00$ & $\$ 119.789 .382,40$ \\
\hline Caso 4 & 53790 & $\$ 270.106 .375,80$ & $\$ 154.535 .770 ; 20$ \\
\hline
\end{tabular}

Fuente: elaboración propia

\subsubsection{Variación capacidad centros de trabajo}

El beneficio total obtenido se ve afectado por la capacidad de minutos ordinarios y extras disponibles en los centros de trabajo CT1 y CT5, y se convirte en un recurso crítico que limita la capacidad del sistema. Este análisis tiene como objetivo, entonces, plantear un nuevo escenario que permita observar y analizar la variación de la función objetivo cuando la capacidad de minutos ordinarios y minutos extras disponibles aumenta en los centros de trabajo que son cuello de botella (CT1 y CT5). Los centros de trabajo CT1 y CT5 limitan al sistema; esto se debe a que todas las prendas a fabricar sin excepción deben pasar por corte (CT1); de allí que su capacidad represente recurso crítico. Por otro, lado aunque no todas las prendas requieren ser estampadas, los resultados obtenidos en el caso de estudio de las variables $Q O$ (familias a producir en taller propio en jornada ordinaria) y $Q E$ (familias a producir en taller propio en jornada extra) sí requieren la utilización de este centro de trabajo, por lo que la utilización de su capacidad aumenta hasta consumir este recurso por completo (ver tabla 9).

Tabla 9. Resultados variación capacidad

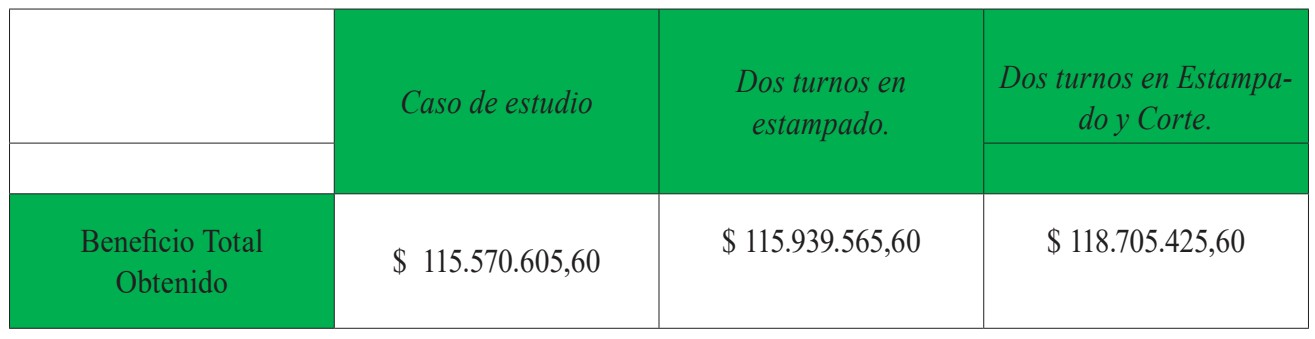

Fuente: elaboración propia

\subsection{Análisis de sensibilidad modelo fase II}

En este apartado se especifican los trabajos a secuenciar, representados por los resultados de las familias a fabricar en el taller propio en los minutos ordinarios disponibles, 
así como el tiempo total que demora cada familia o trabajo en cada centro de trabajo, obtenido al multiplicar la cantidad de prendas en cada familia por el tiempo unitario de cada una de ellas para cada centro. Al insertar los datos en el modelo, se obtuvo como resultado un make span de 34.064,7 minutos; la secuencia de fabricación de cada uno de los trabajos, así como el tiempo que debe esperar cada trabajo para ser procesado en el siguiente centro de su secuencia se muestran en la tabla 10.

Tabla 10. Resultados modelo Scheduling

\begin{tabular}{|c|c|c|c|c|c|c|c|}
\hline \multicolumn{2}{|c|}{ MAKESPAN } & \multicolumn{5}{c|}{34064,7} \\
\hline \multirow{2}{*}{$\begin{array}{c}\text { Posición de la } \\
\text { secuencia }\end{array}$} & \multirow{2}{*}{ Trabajo } & \multicolumn{7}{|c|}{ Tiempo que espera cada trabajo en cada centro para ser procesado } \\
\cline { 4 - 8 } & & CT1 & CT2 & CT3 & CT4 & CT5 & CT6 \\
\hline 1 & 8 & 0 & 0 & 0 & 0 & 0 & 0 \\
\hline 2 & 2 & 0 & 0 & 0 & 0 & 0 & 0 \\
\hline 3 & 4 & 2796,7 & 10675 & 0 & 2245 & 1990 & 0 \\
\hline 4 & 1 & 4289,9 & 12163,3 & 0 & 1835 & 3507,5 & 0 \\
\hline 5 & 15 & 4221,6 & 12148,3 & 0 & 2225 & 3482,5 & 0 \\
\hline 6 & 10 & 3856,6 & 11783 & 0 & 1835 & 3507,5 & 0 \\
\hline 7 & 6 & 4243,9 & 12168,7 & 0 & 1833 & 3893,5 & 0 \\
\hline 8 & 9 & 1123,7 & 9111 & 0 & 0 & 220,5 & 0 \\
\hline 9 & 5 & 18345,9 & 0 & 0 & 442,7 & 1131 & 0 \\
\hline 10 & 3 & 18058,8 & 0 & 0 & 0 & 789 & 0 \\
\hline 11 & 7 & 18407 & 0 & 0 & 314 & 815 & 0 \\
\hline 12 & 13 & 18444,3 & 55,3 & 0 & 372 & 826 & 0 \\
\hline 13 & 14 & 18156,3 & 0 & 0 & 37 & 427 & 0 \\
\hline 14 & 12 & 18437,2 & 261 & 141 & 138 & 506,8 & 0 \\
\hline 15 & 11 & 18268,4 & 0 & 0 & 0 & 220,3 & 0 \\
\hline
\end{tabular}

Fuente: elaboración propia

La capacidad en minutos de todo el sistema en un mes es de 14.664 minutos, lo cual varía para cada centro de trabajo de acuerdo con el número de recursos disponibles, donde se esperaría, entonces, que el valor del make span estuviera cerca a ese valor. En este orden de ideas, el resultado óptimo del make span que arroja el modelo es más grande que el valor esperado; porque el modelo de planeación propuesto inicialmente al ser en esencia un plan agregado no considera las precedencias, ni el tiempo que deben esperar los trabajos en cada centro de trabajo mientras que un trabajo está siendo procesado; por lo tanto, se deben hacer ajustes en el modelo de planeación para que 
el resultado del make span esté cerca al valor esperado. Para esto se consideraron las ecuaciones sugeridas por [2] que aparecen especificadas en la sección 2.5 obteniéndose así un factor de utilización de 0.31 .

$\mathrm{Al}$ afectar los tiempos de procesamiento de cada familia en cada centro de trabajo, el beneficio total obtenido disminuye con base en el resultado inicial del modelo; $\$$ $115.570 .605,6$ y $\$ 87.625 .375,6$, respectivamente, para cada caso. Los cambios en el beneficio se deben a que las cantidades a realizar dentro del taller en minutos ordinarios disminuyeron, ya que los tiempos de procesamiento aumentaron. El valor de make span disminuye significativamente con relación al obtenido inicialmente, donde se presenta una utilización de la capacidad del $85.4 \%$, pasa de $34.064,7$ a 12.526 minutos, respectivamente.

\subsection{Resultado modelo Scheduling con ajuste capacidad total}

Al alterar la restricción de capacidad del centro de trabajo en el taller propio, el beneficio total obtenido disminuye con base en resultado inicial del modelo; $\$ 115.570 .605,6$ y $\$$ 101.635.093,6, respectivamente, para cada caso. El valor de make span disminuye significativamente con relación al obtenido inicialmente, donde se presenta una utilización de la capacidad del $95.1 \%$, al pasar de 34.064,7 a 13.945,9 minutos, respectivamente. Esta solución resulta ser mejor que la anterior debido a que se presenta un mayor porcentaje de utilización y este valor se encuentra más cercano al valor esperado (ver tabla 11).

Tabla 11. Resultado modelo Scheduling ajuste capacidad en cada centro trabajo

\begin{tabular}{|c|c|c|c|c|c|c|c|}
\hline \multicolumn{2}{|c|}{ MAKESPAN } & \multicolumn{6}{c|}{13945,9} \\
\hline $\begin{array}{c}\text { Posición } \\
\text { de la }\end{array}$ & \multirow{2}{*}{$\begin{array}{c}\text { Trabajo } \\
\text { secuencia }\end{array}$} & & \multicolumn{2}{|c|}{ Tiempo que espera cada trabajo en cada centro para ser procesado } \\
\cline { 4 - 8 } & $C T 1$ & $C T 2$ & $C T 3$ & $C T 4$ & CT5 & CT6 \\
\hline 1 & 3 & 0 & 0 & 0 & 0 & 0 & 0 \\
\hline 2 & 6 & 0 & 3223,3 & 0 & 0 & 0 & 0 \\
\hline 3 & 16 & 2732,1 & 0 & 0 & 0 & 0 & 0 \\
\hline 4 & 2 & 3027,8 & 318,3 & 0 & 325 & 7664 & 0 \\
\hline 5 & 9 & 0 & 0 & 0 & 0 & 0 & 0 \\
\hline 6 & 5 & 1761,5 & 1380,5 & 0 & 3165,5 & 892,5 & 0 \\
\hline 7 & 10 & 2932,5 & 1628 & 0 & 1386 & 1386 & 0 \\
\hline 8 & 4 & 2256,9 & 0 & 0 & 0 & 0 & 0 \\
\hline 9 & 13 & 3712,1 & 1368 & 0 & 1632 & 156 & 0 \\
\hline
\end{tabular}




\begin{tabular}{|c|c|c|c|c|c|c|c|}
\hline \multicolumn{7}{|c|}{ MAKESPAN } & \multicolumn{7}{c|}{ 13945,9 } \\
\hline \multirow{2}{*}{$\begin{array}{c}\text { Posición } \\
\text { de la } \\
\text { secuencia }\end{array}$} & \multirow{2}{*}{ Trabajo } & \multicolumn{2}{|c|}{ Tiempo que espera cada trabajo en cada centro para ser procesado } \\
\cline { 5 - 9 } & CT1 & CT2 & CT3 & CT4 & CT5 & CT6 \\
\hline 10 & 12 & 6995,2 & 0 & 0 & 0 & 6 & 0 \\
\hline 11 & 8 & 7483,7 & 0 & 0 & 0 & 1,5 & 0 \\
\hline 12 & 14 & 7519,6 & 109 & 0 & 0 & 10,5 & 0 \\
\hline 13 & 15 & 7521,9 & 110,3 & 0 & 4 & 8 & 0 \\
\hline 14 & 1 & 7520,5 & 11,3 & 0 & 0 & 3 & 0 \\
\hline 15 & 7 & 7635,4 & 0 & 0 & 4 & 5 & 0 \\
\hline 16 & 11 & 7637 & 0 & 8 & 0 & 0 & 0 \\
\hline
\end{tabular}

Fuente: elaboración propia

\section{CONCLUSIONES}

Este modelo se convierte en una herramienta eficiente de planeación que permite tomar decisiones referentes en ofrecer el servicio de maquila a otras empresas, programar más de un turno para los centros de trabajo que restringen el sistema, comprar maquinaria para aumentar la capacidad de los centros de trabajo y programar el inicio de las operaciones de dichos centros de trabajo más temprano que los demás de tal forma que se logre disminuir tiempo de inactividad, entre otras.

Analizar el comportamiento del modelo cuando varía la demanda de las familias, con el propósito de ver el impacto de las modificaciones en la función objetivo y en las variables de interés, se encontró que un aumento en la demanda provoca un aumento en la función de desempeño creciente, pero no proporcional, ya que la capacidad de producción se encuentra limitada por algunos centros de trabajo que representan el cuello de botella del sistema. Se identificaron centros de trabajo que representan una limitante al sistema, representados por corte (CT1) y estampado (CT5).

Asímismo, se realizó un análisis del comportamiento de la función objetivo del modelo al variar las capacidades limitantes de estos centros identificados, dando como resultado un aumento del $2.78 \%$ frente a los resultados iniciales obtenidos en el caso de estudio, que responden a un aumento al doble de la capacidad inicial de estos centros representada por adquirir una unidad de maquinaria de más para cada uno de ellos; se da como resultado la no utilización de minutos extra ya que se cuenta con la capacidad suficiente para satisfacer la demanda produciendo en minutos ordinarios disponibles.

Por otro lado, al analizar los resultados arrojados por el modelo fase 2, se puede observar que el modelo fase 1 no tiene en cuenta factores importantes como son los 
tiempos de espera en cada centro, ni las precedencias relacionadas a cada una de las familias de productos al ser su resultado información de entrada del modelo de Scheduling; este arroja un valor del make span mayor que el tiempo total disponible al mes, es decir, los pedidos no van a poder ser entregados en el tiempo acordado. Se hizo necesario realizar modificaciones al modelo fase $1 \mathrm{o}$ a los datos de entrada de dicho modelo para obtener un resultado de acuerdo con la capacidad real del sistema en el horizonte de plan definido; para este caso se tomaron en cuenta los factores de utilización para cada centro de trabajo y la utilización total del sistema; de acuerdo con los análisis de los resultados, la mejor solución es la de modificar la restricción de capacidad de minutos ordinarios del modelo fase 1 con el cual se obtuvo el mayor porcentaje de utilización $95 \%$, respectivamente. Aunque el modelo aún no ha sido implementado, de acuerdo con los resultados obtenidos cuando fue ejecutado con los datos del caso de estudio se evidencia que es una herramienta eficiente de gestión de producción que permitiría a la empresa mejorar su nivel de respuesta frente a los clientes, tener una política de distribución de las cantidades a producir en el taller propio y enviar a maquila de tal forma que se perciba un mayor beneficio económico, y a su vez disminuir los costos de contratación del personal, ya que sólo se contratarían los operarios requeridos en cada centro de trabajo para cumplir con la demanda de un mes.

Por otra parte, es importante mencionar que para la implementación del modelo de planeación dentro de cualquier organización se requiere desarrollar un aplicativo de software o herramienta de gestión del modelo, como la propuesta realizada por [12]. Finalmente, los modelos de programación matemática permiten, mediante su resolución, ayudar a la toma de decisiones generando soluciones óptimas, dado un objetivo establecido. Asímismo, pueden ser utilizados para evaluar el impacto de tomar decisiones, antes de implementarlas, y de este modo elegir la que más se ajuste a la solución requerida.

\section{REFERENCIAS BIBLIOGRÁFICAS}

[1] D. Sipper and R.L Bulfin, "Planeación y Control de la Producción”. México: McGraw-Hill, 1998. 175.

[2] J. A. Domínguez Machuca, "Dirección de Operaciones, Aspectos Tácticos y Operativos en la Producción y los Servicios”. España: McGraw-Hill, 1995. 37.

[3] M. L. Pinedo, "Scheduling. Theory, Algorithms, and Systems". Estados Unidos. Prentice Hall, 2012. 676 .

[4] P. Egri, A. Kovács, A Marcus and J Vánza, "Project-Oriented approach to production planning and scheduling in Make to Order Manufacturing", Production Systems and Information Engineering, vol. 2, No. 1, Diciembre 2004, pp. 22-36. 
[5] M Gutiérrez Fernández, "Rediseño de procesos del sistema de planificación y control de la producción en la industria de ingeniería - bajo- pedido basado en las tecnologías de la información”. Tesis Doctoral. Universidad Politécnica de Madrid, 2009.

[6] J. P. Orejuela Cabrera, J. J. Ocampo Carrillo and C. A. Micán Rincón, "Propuesta metodológica para la programación de la producción en las PYMES del sector artes gráficas, área Publi-Comercial", Estudios Gerenciales, vol. 26, No. 2, Enero 2010, pp. 97-118.

[7] L. Giraldo Páez and M Salcedo Galíndez "Propuesta metodológica para la estimación de la capacidad de la producción en un restaurante de fabricación masiva”. Tesis Ingeniería Industrial. Universidad del Valle, 2010.

[8] N. Núñez Orozco and W. F. Arroyave "Planeación y control de la producción en ambientes de fabricación Make to Order". Tesis Ingeniería Industrial. Universidad del Valle, 2011.

[9] S. Ruiz, O. D. Castrillón and W. A. Sarache, "A Multiobjective Methodology to Optimize a Job Shop Environment”, Información Tecnológica, vol. 23, No. 1, Abril 2011, pp. 35-46.

[10] P. Escobar, J. A. Giraldo and D. M. Cárdenas, "Scheduling Hybrid Production Systems, Make to Stock/ Make to Order, using Group Analytic Hierarchy Process Ordering (GAHPO)", Información Tecnológica, vol. 23, No. 5, Mayo 2012, pp. 33-46.

[11] J. P. Orejuela Cabrera "Desarrollo de un modelo jerárquico de planeación de la producción en un flowshop: Caso Industria de Concentrados”. Tesis Ingeniería Industrial. Universidad del Valle, 2008.

[12] H. H. Toro Díaz y L. Delgado Hidalgo, “Optimizing a two-echelon capacity-constrained material requirement manufacturing system using a linear programming model”, Ingeniería e Investigación, vol. 30, No. 1, Abril 2010, pp. 168-173. 